\title{
The Michigan 10-Degree Blue Spectral Survey as a Basis for Future Deep Schmidt Surveys
}

\author{
N. Houk \\ University of Michigan, USA
}

\section{T. von Hippel}

University of Cambridge, Institute of Astronomy, UK

\begin{abstract}
The Henry Draper stars are being systematically classified on the MK System, using the Curtis and Burrell Schmidt telescopes with photographic spectra having a dispersion of $108 \AA / \mathrm{mm}$. Over 156,000 stars south of $\delta=+5^{\circ}$, have been classified leaving about 69,000 yet to do. The project is expected to be completed around the year 2004 . This all-sky network of consistently classified spectra of very good quality should serve as a basis for future deep surveys. Such surveys will almost certainly be automated because of the huge number of stars to be dealt with. Von Hippel et al. at Cambridge plan to scan at least 150,000 of the spectra classified by Houk, using her plates to serve as a 'training' set for automatic classification using artificial neural networks. The same data can also be utilized for other methods of automatic classification including the metric-distance methods used by Kurtz and La Sala (Kurtz 1983). Even at lower dispersions, significantly more information can be obtained from Schmidt spectra than by doing Schmidt photometric colour surveys alone, though these are also valuable, especially when used in conjunction with spectra. We urge that large Schmidts not currently having prisms or other dispersive elements consider adding this equipment.
\end{abstract}

The spectra that I'll be talking about today were taken with the Curtis and Burrell Schmidt telescopes, respectively at Cerro Tololo, Chile and Kitt Peak, Arizona, using $10^{\circ} \mathrm{objective-prism} \mathrm{combinations} \mathrm{giving} \mathrm{a} \mathrm{dispersion} \mathrm{of} 108$ $\AA / \mathrm{mm}$, and the spectra were well-widened to $0.8 \mathrm{~mm}$. Since many of you don't work with spectra, I'll pass around copies of our Second Objective-Prism Atlas (Houk, Newberry 1984), with candidates selected from our $10^{\circ}$ blue survey plate collection published in 1984. This should give you an idea of the high quality of the spectra, which is better than for many slit spectra.

Our $10^{\circ}$ blue survey used IIaO plates, and, fortunately, plate-taking for the entire sky was completed about $11 / 2$ years ago, before these plates became unavailable from Kodak. Two plates or more were taken of each $5^{\circ} \times 5^{\circ}$ field, a $20^{m}$ exposure and a $1^{m}+4^{m}$ exposure to get the whole range of magnitudes from about fifth to tenth or eleventh (blue).

Visual spectral classification of over 150,000 of the HD stars (Houk, Cowley 1975; Houk 1978; Houk 1983; Houk, Smith-Moore 1988) is clearly the last gasp of the visual classifying mode, for surveys, at least. The next generation, dealing 
with huge amounts of data, will obviously have to use automated methods. Don't underestimate the power of the human eye/brain combination, however. With experience the eye can detect very small differences in line strengths and shapes, line ratios, etc. It is great at pattern recognition. As I classify, I am not just looking at specific lines and criteria, but I am looking at the whole general appearance of the spectrum and how closely it matches the entire blue spectrum of a standard star. Working with much lower dispersion spectra during my graduate student and postdoc days at Case Warner and Swasey Observatory in Cleveland undoubtedly helped instill this approach. By the way, Bambang Hidayat was a fellow graduate student with me there, and I'm very happy to have finally had the chance to visit him and his observatory here in Indonesia. About 10 per cent of the stars are classified independently on more than one plate, and internal rms errors calculated from these are $\sim 0.6$ spectral subtypes and $\sim 0.25$ luminosity classes. About half the spectra are compared directly with standards during classification.

You may have noticed in the poster paper by Houk et al. (1995, these proceedings) that only surface distributions of the stars of various types in Aitoff charts by galactic coordinate were displayed without even attempting to calculate distances. In some earlier work, mostly unpublished, stars with good photoelectric magnitudes were used to try to correct the HD magnitudes, which were systematically off for the fainter stars. However, large random scatter remained, being as great as a full magnitude even for the brighter stars from 6th to 9th magnitude (blue). I mention this because astronomers now seem to be concentrating on much fainter stars, but there is still much to be done on the apparently brighter stars to help us with galactic structure studies, etc.

To get back to the Michigan Survey, Table 1 shows what we have done so far and rough estimates for our future timetable. We expect Volume 5, which will include HD stars from $\delta=-12^{\circ}$ to $+5^{\circ}$, to be finished by the end of 1994 , in machine readable form. However, producing the hard copy takes time and it may not be out until sometime in the first half of 1995 . One thing that is slowing us down at the moment is that the University of Michigan is phasing out its mainframe computer, and we are having to re-write all our programs and procedures for producing the volume using UNIX and the Sun work stations, with disk storage instead of magnetic tapes.

\begin{tabular}{rrrrr}
\hline Volume & Declination & No. of HD Stars & Running Total Classified & Year of Publ. \\
\hline 1 & $-90^{\circ}$ to $-53^{\circ}$ & 36,382 & 36,382 & 1975 \\
2 & $-53^{\circ}$ to $-40^{\circ}$ & 30,400 & 66,782 & 1978 \\
3 & $-40^{\circ}$ to $-26^{\circ}$ & 30,314 & 97,096 & 1982 \\
4 & $-26^{\circ}$ to $-12^{\circ}$ & 33,301 & 130,397 & 1988 \\
5 & $-12^{\circ}$ to $+5^{\circ}$ & $\sim 32,500$ & $\sim 162,900$ & $1994 / 5$ \\
$6 \& 7$ & $+5^{\circ}$ to $+90^{\circ}$ & $\sim 62,400$ & 225,300 & $\sim 1999, \sim 2004$ \\
& $(2$ volumes) & & & \\
\hline \hline
\end{tabular}

This table also shows clearly the predominance of southern stars in the Henry Draper Catalogue. Only two volumes will be required for all the HD stars north of $+5^{\circ}$. So with completion of Volume 5 , only about $1 / 4$ of the stars will remain to be classified. 
Turning to the future, I want to emphasize the importance of other astronomers continuing to do spectral and photometric surveys. Without going into detail, I'd just like to point out that we do need surveys of fainter stars. As Garrison and others have emphasized, we need to have fainter standard stars, because most of the larger telescopes have a hard time dealing with very bright stars. The Michigan catalogues have been of some help in the 6-10 blue magnitude range but to get the fainter standards, say around 14th magnitude, we have to look at a lot of candidates. Even at lower dispersions, significantly more information can be obtained from Schmidt spectra than by doing Schmidt photometric color surveys alone, though these are also valuable, especially when used in conjunction with spectra. We urge that astronomers having large Schmidt telescopes consider adding prisms or other dispersive elements if they are not currently being used.

You may have noticed that Ted von Hippel is listed as a co-author of this paper. He was unable to be here so I'm reporting orally on some of his and other's work on automated classification of stellar spectra. This will not be included in the written proceedings since most of it is published in more extensive form in the Proceedings of the Workshop held in Tucson in September 1993 celebrating the 50th anniversary of Morgan and Keenan's system of classification. See Houk's paper titled The Michigan Survey and the Continuing Importance of Surveys(Houk, 1994) and the paper by Ted von Hippel et al.Automated Classification of Stellar Spectra: Where are we now? (von Hippel et al. 1994a) Also related is a paper to be published soon in MNRAS, Automated Classification of Stellar Spectra I: Initial Results with Artificial Neural Networks (von Hippel et al. 1994b).

\section{Acknowledgements}

N. Houk wishes to thank the National Science Foundation of the U.S.A. for its continuing support.

\section{References}

Houk N. \& Cowley A. P., 1975,Michigan Catalogue of Two-dimensional Spectral Types for the HD Stars, Vol.1, Dept. of Astronomy, University of Michigan, Ann Arbor

Houk N., 1978, Michigan Catalogue of Two-Dimensional Spectral Types for the HD Stars, Vol. 2, Dept. of Astronomy, University of Michigan, Ann Arbor

Houk N., 1982, Michigan Catalogue of Two-Dimensional Spectral Types for the HD Stars, Vol. 3, Dept. of Astronomy, University of Michigan, Ann Arbor

Houk N. \& Newberry, M., 1984, A Second Atlas of Objective-Prism Spectra,Dept. of Astronomy, University of Michigan, Ann Arbor

Houk N. \& Smith-Moore M., 1988, Michigan Catalogue of Two-Dimensional Spectral Types for the HD Stars, Vol. 4, Dept. of Astronomy, University of Michigan, Ann Arbor 
Houk N., 1994, ASP Conf. Ser. 60, C. J. Corbally, R. O. Gray \& R. F. Garrison, eds, p. 285

Kurtz M. J., 1983, The MK Process and Stellar Classification, R. F. Garrison, ed., (David Dunlop Obs, Toronto), p. 136

von Hippel T., Storrie-Lombardi L. J., Storrie-Lombardi M. C. \& Irwin M. J., 1994, ASP Conf. Ser. 60, C. J. Corbally, R. O. Gray \& R. F. Garrison

von Hippel T., Storrie-Lombardi L.J., Storrie-Lombardi M.C. \& Irwin M. J. 1994, MNRAS, submitted

\section{Discussion}

Kurtanidze: How large are the differences in the completeness limiting magnitude for stars of different spectral types? Do they also depend on luminosity classes?

Houk: The limiting magnitude does vary somewhat from plate to plate. Certainly the limiting magnitude does reduce the number of late $(K, M)$ dwarfs we find.

Raharto: What is the main reason for the difficulty in obtaining the standard spectra of stars with spectral classes later than M4?

Houk: We have measured only six plates at this point and these didn't have examples of stars ealier than B3 and later than M4. When more plates are measured, there will be enough examples to extend this range.

Cannon: I don't understand why von Hippel has to make a visual inspection to spot overlapping spectra. We know from positional catalogues where all the stars are, so it is possible to predict overlaps. Secondly, in our low dispersion work with the UK Schmidt, we found we could solve a lot of the problems by taking pairs of prism plates with perpendicular directions of dispersion.

Houk: He is just starting and I will give your first suggestion to him. Early in our plate taking we tried a rotated prism, but found that our spectra were then usually overlapped with something else, so we didn't do much of this. Of course, our spectra are consistently longer than your lower-dispersion ones. Overlapping is not a problem for me, doing visual classification. 